\title{
Quarkonium production at the Fermilab Tevatron through soft color interactions
}

\author{
A. Edin \\ Department of Radiation Sciences, Uppsala University, Box 535, S-751 21 Uppsala, Sweden \\ G. Ingelman \\ Department of Radiation Sciences, Uppsala University, Box 535, S-751 21 Uppsala, Sweden \\ and Deutsches Elektronen-Synchrotron DESY, Notkestrasse 85, D-22603 Hamburg, Germany \\ J. Rathsman \\ Stanford Linear Accelerator Center, Stanford University, Stanford, California 94309
}

(Received 15 May 1997)

\begin{abstract}
The direct charmonium and bottomonium production rate observed at high $p_{\perp}$ in $p \bar{p}$ collisions at the Fermilab Tevatron is factors of 10 larger than predictions based on conventional perturbative QCD. We show that this excess can be accounted for by our model for soft color interactions, previously introduced to describe in a novel way the large rapidity gap events observed at DESY HERA. [S0556-2821(97)06623-X]
\end{abstract}

PACS number(s): 13.60.Le, 12.38.Aw, 13.85.Ni, 24.10.Lx

Heavy quarkonia, i.e., bound states of heavy-quarkantiquark pairs, are thought to provide a useful testing ground for perturbative quantum chromodynamics (PQCD), the reason being that the charm and bottom quark masses provide a large scale which makes the short distance behavior calculable using a perturbative approach whereas the nonperturbative contributions can be factorized into a wave function. It was therefore a surprise that measurements by the Collider Detector at Fermilab (CDF) [1] and D $\emptyset$ [2] Collaborations at the Fermilab Tevatron $p \bar{p}$ collider $(\sqrt{s}=1.8$ $\mathrm{TeV})$ gave cross sections of direct high- $p_{\perp} J / \psi, \psi^{\prime}$ and $Y, Y^{\prime}$ production far above the expectation from the color singlet model [3] based on conventional PQCD. The observed excess is generally an order of magnitude and increases to a factor $\sim 50$ with increasing $p_{\perp}$.

In the color singlet model PQCD is used to calculate the production of a $Q \bar{Q}$ pair in a color singlet state. This forms a quarkonium state with the same angular momentum quantum numbers ${ }^{2 S+1} L_{J}$ by coupling to the nonperturbative wave function at the origin, which is obtained from the leptonic decay width. The striking failure of this model has led to several phenomenological investigations and some new models; for a review see [4]. The color octet model, which is based on non-relativistic QCD, also takes into account the more abundant perturbative production of $Q \bar{Q}$ pairs in color octet states. The unknown probability for the transformation into color singlets due to nonperturbative processes is parametrized with matrix elements that have to be fitted to data, but are universal so that the model can be tested by studying several different processes. Similarly, in the color evaporation model [5] a certain fraction of all $Q \bar{Q}$ pairs, independently of their production process and quantum numbers, form a quarkonium state. Thus, both the color octet model and the color evaporation model requires fitting to experimental data. In contrast, our soft color interaction (SCI) model [6] gives a prediction also of the absolute rate which, as will be shown in this paper, is in good agreement with the Tevatron data.
The SCI model was introduced as a novel way to interprete the rapidity gap events observed in deep inelastic scattering (DIS) at the DESY ep collider HERA. The model does indeed describe the salient features of the ZEUS [7] and H1 [8] data. The conventional interpretation of these events is in terms of deep inelastic diffractive hard scattering [9] on partons in the Pomeron, which is a colorless object exchanged from the quasielastically scattered proton. Although phenomenological models based on this idea work quite well to describe the data, there are theoretical problems with the Pomeron approach. In particular, the factorization into a Pomeron flux, a Pomeron parton density, and a hard interaction described by a QCD matrix element may not be universal for all processes, e.g., DIS and hadron collisions. The Pomeron-proton interaction is soft and thereby occurs on a long space-time scale. It may therefore be incorrect to consider the Pomeron as decoupled from the proton during and after the hard scattering.

It is more natural to expect soft interactions with the proton both before and after the snapshot of the DIS probe. To investigate this line of thinking, we have developed a model [6] with a mechanism for soft color interactions as an alternative to the approach based on the Pomeron and Regge phenomenology.

The basic idea in our SCI model is that there may be additional soft interactions, not previously accounted for, at a scale below the cutoff $Q_{0}^{2}$ for perturbative QCD. Obviously, interactions do not disappear below this cutoff. The question is how to take them into account properly and whether conventional hadronization models give a complete description. We propose that the quarks and gluons produced by conventional PQCD processes, as described by matrix elements and parton showers, interact nonperturbatively with the color medium of the proton remnant. This should be a natural part of the process in which "bare" perturbative partons are "dressed" into nonperturbative ones and the formation of the confining color flux tube in between them. These soft interactions cannot change the momenta of the partons significantly, but they change their color and thereby affect the 

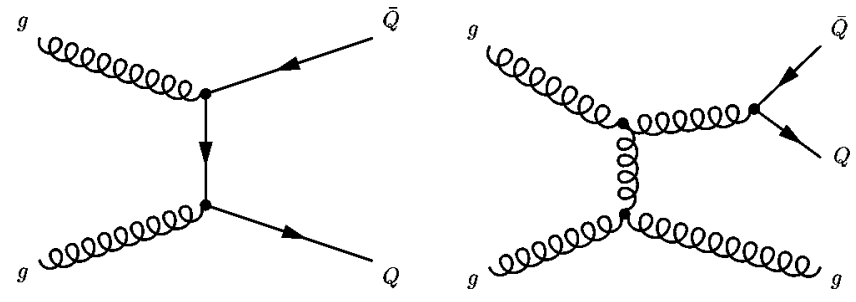

FIG. 1. Main PQCD processes for production of a $Q \bar{Q}$ pair in (a) leading order and (b) next-to-leading order.

color structure of the event. This in turn will lead to a modified hadronic final state, e.g., with rapidity gaps or quarkonium.

Lacking a proper understanding of nonperturbative QCD processes, we have constructed a model [6] to describe and simulate such soft color interactions within conventional Monte Carlo (MC) event generators. All partons from the hard interaction plus the remaining partons in the proton remnant are assigned appropriate color and anticolor charges. Partons from the hard interaction can make a soft interaction with partons in the proton remnant exchanging their colors but not changing their momenta, which may be viewed as soft nonperturbative gluon exchange. Similarly, partons in the proton remnant can exchange color whereas no direct color exchanges are allowed between the perturbative partons. Since the process is nonperturbative the exchange probability for a pair cannot be calculated from first principles and is instead described by a phenomenological parameter $R$. The number of soft exchanges will vary event-by-event and change the color topology. In the Lund string model [10] this corresponds to a modified string stretching that alters the outcome of the hadronization.

The SCI model was first implemented in LEPTO [11] simulating DIS. Here, the main effect producing rapidity gap events at HERA is SCI between the partons from the hard scattering and those in the proton remnant. In particular, with gluon initiated processes at small Bjorken- $x$, the color octet charge of the produced hard parton system ( $q \bar{q}$ plus possible additionally emitted partons) can turn into a color singlet. This may give a hadronic system $X$ separated in rapidity from a very forward moving proton remnant system of small mass (e.g., a proton or a resonance). The main features of diffractive scattering emerges naturally and the HERA rapidity gap events can be surprisingly well described by this simple model [6].

This SCI model can be straightforwardly applied to describe heavy-quarkonium production in $p \bar{p}$; a color octet $Q \bar{Q}$ pair from PQCD can be turned into a singlet and thereby form a quarkonium state, provided that the mass is appropriate. In leading order (LO) PQCD heavy-quark production occurs through $g g \rightarrow Q \bar{Q}$ [Fig. 1(a)] and $q \bar{q} \rightarrow Q \bar{Q}$. However, higher order processes involving gluon splitting $g \rightarrow Q \bar{Q}$ are important. For example, the next-to-leading order (NLO) process $g g \rightarrow g Q \bar{Q}$ illustrated in Fig. 1(b) gives a large contribution because it is an $\alpha_{s}$ correction to the large cross section for gluon scattering $(g g \rightarrow g g)$. Matrix elements with explicit heavy-quark masses are available up to NLO. However, since the virtuality of the gluon need not be very large to split, in particular to $c \bar{c}$, still higher orders may be important at Tevatron energies. These can be taken into account approximately through the parton shower approach.

The LO and parton shower production of $Q \bar{Q}$ pairs are available in the MC generator PYTHIA [12]. On the generated parton level events we apply the above SCI mechanism which will turn some $Q \bar{Q}$ pairs into color singlets. A quarkonium state is then produced if the invariant mass $m_{Q \bar{Q}}$ is below threshold for open heavy flavor production $\left(2 m_{M}\right)$. Thus, the cross section is

$$
\sigma_{\text {quarkonium }}=\int_{2 m_{Q}}^{2 m_{M}} \frac{d \sigma^{1}}{d m_{Q \bar{Q}}} d m_{Q \bar{Q}},
$$

where the singlet $Q \bar{Q}$ cross section $d \sigma^{1}=d \sigma \otimes \mathrm{SCI}$ is obtained from the application of the SCI model on PYTHIA events. This is not just a constant fraction of the original $Q \bar{Q}$ cross section, but depends somewhat on the partonic state and the possible string configurations. Whereas the number of possible string configurations increases with parton multiplicity, the number of string configurations giving a singlet $Q \bar{Q}$ pair is more or less constant. Therefore, the relative probability for a singlet $Q \bar{Q}$ decreases slowly with parton multiplicity. This in turn, implies a slight decrease $(\sim 10 \%$ in the observed $p_{\perp}$ range) of the singlet $Q \bar{Q}$ fraction with increasing $p_{\perp}$ in the hard scattering process which causes more abundant parton showering.

The invariant mass of the $Q \bar{Q}$ pair is in principle given by the PQCD process. However, we expect this mass to be smeared by the soft color interactions involving energymomentum transfers of the order a few hundred $\mathrm{MeV}$. Irrespective of the original $Q \bar{Q}$ mass (below $2 m_{M}$ ), we therefore divide the quarkonium cross section onto the different quarkonium states based simply on spin statistics as suggested by [5]. This should be a good approximation since the heavy-quark system is nearly nonrelativistic. The cross section for a given quarkonium state $X$ with total angular momentum $J_{X}$ is then given by

$$
\sigma_{X}=\frac{\Gamma_{X}}{\sum_{Y} \Gamma_{Y}} \sigma_{\text {quarkonium }},
$$

where $\Gamma_{X}=\left(2 J_{X}+1\right) / n_{X}$ corresponds to a partial width. Here, we have included a suppression of radially excited states, i.e., with the main quantum number $n_{X}$. This reproduces approximately the differences between different $\psi$ and $Y$ states regarding their leptonic width and thus the wave function at the origin [13], which should be particularly relevant when the essentially pointlike $Q \bar{Q}$ pair forms a quarkonium state.

The results of this model are shown in Fig. 2 and Table I together with the Tevatron data. The agreement is remarkably good, considering the simplicity of the SCI model and the fact that it was originally contructed for a different physics issue. As mentioned, the absolute normalization is not adjusted to data, but given by the model. The only parameter in the SCI model, i.e., the probability $R$ of a color exchange between two partons, is kept at the value $R=0.5$ chosen to reproduce the rate of rapidity gap events in DIS at HERA. However, as for the rapidity gap rate, the dependence of the 


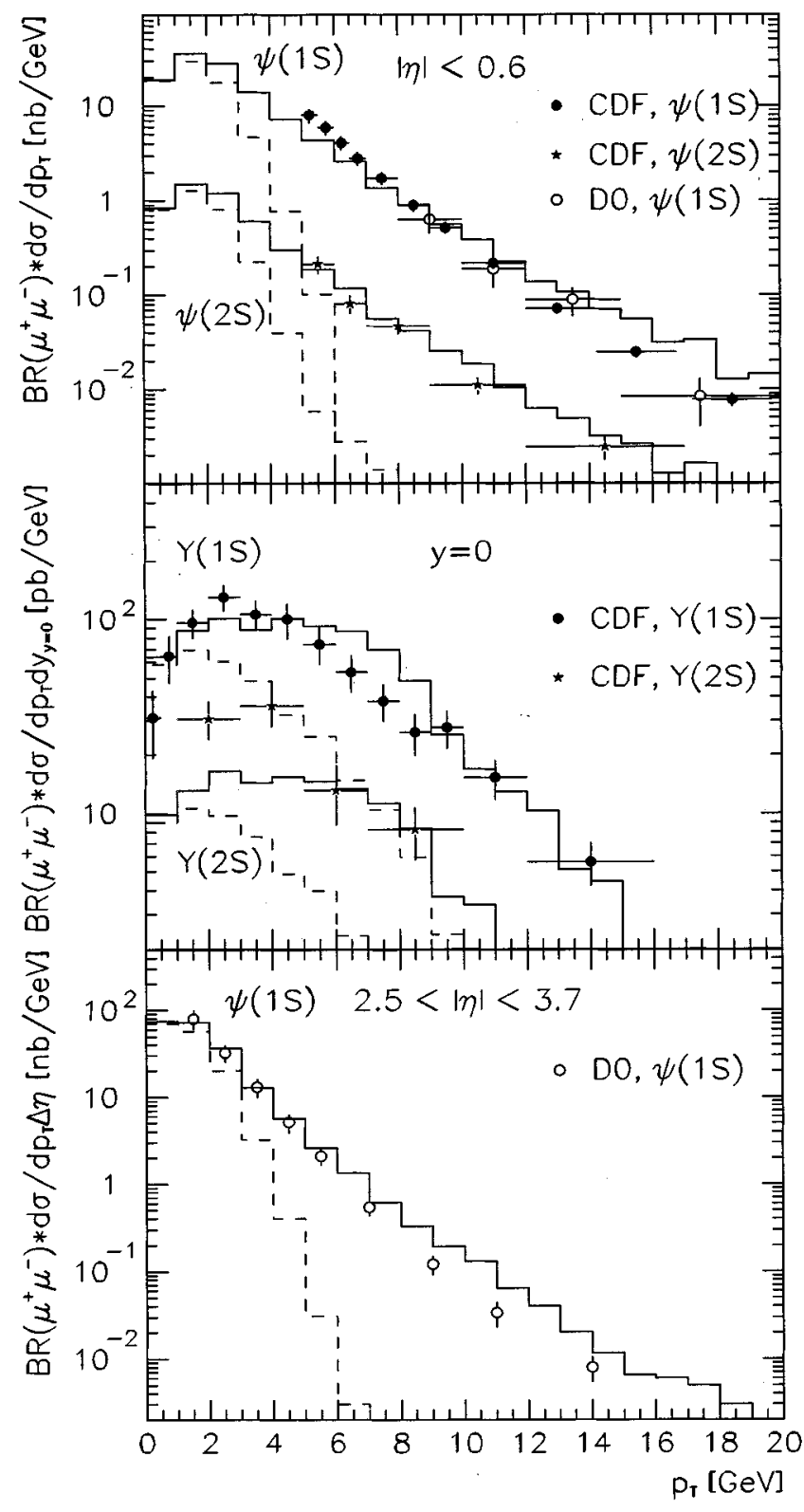

FIG. 2. Transverse momentum spectra of prompt charmonium $J / \psi, \psi^{\prime}$ and bottomonium $Y, Y^{\prime}$ in $p \bar{p}$ collisions at the Tevatron energy. Data from CDF [1] (filled symbols) and DФ [2] (open symbols) compared to results from the soft color interaction (SCI) model applied to $c \bar{c} / b \bar{b}$ production from leading order $\left(\alpha_{s}^{2}\right)$ matrix elements (dashed lines) and with the inclusion of higher order contributions calculated in the parton shower approach (full lines). Production central in pseudorapidity (a) $|\eta|<0.6$, (b) $|y|<0.4$, and forward (c) $2.5<|\eta|<3.7$ (where an assumed $20 \%$ systematic error has been added in quadrature).

quarkonium rate on this parameter is quite small. For example, changing to $R=0.1$ only decreases the cross section with $\sim 30 \%$.

There is, however, a strong dependence on the heavyquark mass $m_{Q}$. We have used $m_{c}=1.35 \mathrm{GeV}$ and $m_{b}=4.8$ $\mathrm{GeV}$. Increasing the quark masses by $0.25 \mathrm{GeV}$ decreases the cross section with a factor 3 , whereas a decrease of the quark masses by the same amount increases the cross section with a factor 2. This is as usual in PQCD heavy-quark production close to threshold and can be understood from Eq. (1).
TABLE I. Relative rates in \% for different quarkonium states in the Monte Carlo model and the Tevatron data [1]. The charmonium cross sections are in general for $p_{\perp} \geqslant 5 \mathrm{GeV}$ and $|\eta|<0.6$ whereas the bottomonium cross sections are for $|y|<0.4$.

\begin{tabular}{lcc}
\hline \hline & MC model & Data \\
\hline$J / \psi$ direct & 61 & $64 \pm 6$ \\
$J / \psi$ from $\chi_{c}$ & 26 & $30 \pm 6^{\mathrm{a}}$ \\
$J / \psi$ from $\psi^{\prime}$ & 13 & $7 \pm 2 \leftrightarrow 15 \pm 5^{\mathrm{b}}$ \\
$\sigma\left(\chi_{c}^{2}\right) /\left(\sigma\left(\chi_{c}^{1}\right)+\sigma\left(\chi_{c}^{2}\right)\right)$ & 62 & $47 \pm 9$ \\
$\sigma\left(\psi^{\prime}\right) / \sigma(J / \psi)$ & 32 & $25 \pm 6^{\mathrm{c}}$ \\
$\sigma(Y(2 \mathrm{~S})) / \sigma(Y(1 \mathrm{~S}))$ & 29 & $46 \pm 9$ \\
\hline
\end{tabular}

${ }^{\mathrm{a} D a t a}$ are for $p_{\perp} \geqslant 4 \mathrm{GeV}$.

${ }^{\mathrm{b}}$ Lower (upper) value for $p_{\perp} \sim 5$ (18) GeV.

${ }^{\mathrm{c}}$ Assuming the same fraction of $J / \psi$ and $\psi^{\prime}$ from $b$ hadron decays.

The cross section also depends somewhat on other features of the Monte Carlo model such as the QCD $\Lambda$ parameter, details in the parton shower model and the choice of parton density parametrizations. These issues are not particular for the SCI model, but of a general character. We therefore do not adjust any of these features, but leave them as they are by default in the PYTHIA MC model which provides a standard for many processes and observables. We note that there are no significant changes in our results when varying the virtuality scale for the parton shower or using different available parton density parametrizations [e.g., MartinRoberts-Stirling (MRS) or CTEQ] based on recent data. However, including the multiple parton-parton interactions in PYTHIA lowers the total quarkonium cross section slightly $(\sim 20 \%)$.

The observed $p_{\perp}$ spectra of the different quarkonium states are also quite well reproduced by the model, as demonstrated in Fig. 2. The contribution from the LO process (shown separately as dashed curves) have a steeper $p_{\perp}$ dependence and only contributes significantly at lower $p_{\perp}$, in particular for $\psi, \psi^{\prime}$. Thus, the higher order contributions are most important.

Comparing model and data in detail, there is a tendency for the model to have a slightly flatter $p_{\perp}$ distribution for the $J / \psi$ production. This may be an effect of using the approximate parton shower gluon splitting $g \rightarrow c \bar{c}$ instead of the NLO matrix element which might be more appropriate at large $p_{\perp}$. It may also be influenced by the detailed contribution of different quarkonium states, since $J / \psi$ from decays contribute more at smaller $p_{\perp}$.

The relative rates of $J / \psi$ from direct production and from decays of higher quarkonium states are given in Table I together with some ratios of cross sections for different quarkonium states. The model gives good agreement with data in most cases. This shows that simple spin statistics can describe the main effects. The only deviation of some significance is the ratio $\sigma(Y(2 S)) / \sigma(Y(1 S))$, where the simple suppression of radially excited states seems too strong.

The SCI model provides a mechanism for how a color octet $Q \bar{Q}$ pair is turned into a singlet and thereby an absolute normalization of the cross section for the quarkonium production. In addition, one obtains a direct relation to other manifestations of soft interactions, such as diffraction and rapidity gaps. In the color octet model the probability to form 
a singlet is parametrized into matrix elements, but no explicit mechanism for how an octet is turned into a singlet is given.

These models are in clear contrast to the color singlet model for quarkonium production which cannot explain the large rate observed at the Tevatron. In addition, they differ in the fragmentation function of $J / \psi$, i.e., $D_{g \rightarrow J / \psi}(z, \mu)$ where $z$ is the momentum fraction of the $J / \psi$ relative to the "mother" gluon and $\mu$ is the factorization scale. In the color singlet model the fragmentation function is relatively flat [14], whereas in the color octet model it is essentially a $\delta$ function at $z=1[15]$.

The fragmentation function can be measured by taking the ratio of the transverse momentum of the $J / \psi$ and the total transverse momentum in a cone centered at the $J / \psi$ [16]. Following conventional jet algorithms for high-energy hadronic collisions, one may choose a cone
$\Delta R=\sqrt{\Delta \phi^{2}+\Delta \eta^{2}} \simeq 0.7$ in pseudorapidity and azimuthal angle. Applying this to our SCI model results in a fragmentation function for $J / \psi$ 's with $p_{\perp}>5 \mathrm{GeV}$ which is strongly peaked at $z=1$. Although its exact form depends on details in the model, it is essentially as expected in the color octet model and very different from the color singlet model.

In summary, we have shown that the large rate of direct high- $p_{\perp}$ charmonium and bottomonium in high-energy $p \bar{p}$ collisions can be quite well described by the soft color interaction model. The same SCI model also accounts for the rapidity gap events observed at HERA. This indicates that these features of nonperturbative strong interactions are quite general and can be described by a universal model.

This work was supported in part by the Swedish Natural Science Research Council and the U.S. Department of Energy, Contract No. DE-AC03-76SF00515.
[1] CDF Collaboration, F. Abe et al., Phys. Rev. Lett. 79, 572 (1997); 75, 4358 (1995); 79, 578 (1997); Report No. FermilabCONF-96/402-E (unpublished).

[2] D0 Collaboration, S. Abachi et al., Report No. FermilabCONF-96/249-E (unpublished).

[3] For references see the review in [4].

[4] E. Braaten, S. Fleming, and T. C. Yuan, Annu. Rev. Nucl. Part. Sci. 46, 197 (1996).

[5] J.F. Amundson, O.J.P. Éboli, E.M. Gregores, and F. Halzen, Phys. Lett. B 372, 127 (1996), and references therein.

[6] A. Edin, G. Ingelman, and J. Rathsman, Phys. Lett. B 366, 371 (1996); Z. Phys. C 75, 57 (1997).

[7] ZEUS Collaboration, M. Derrick et al., Phys. Lett. B 315, 481 (1993); Z. Phys. C 68, 569 (1995).
[8] H1 Collaboration, Nucl. Phys. B429, 477 (1994); Phys. Lett. B 348, 681 (1995).

[9] G. Ingelman and P.E. Schlein, Phys. Lett. 152B, 256 (1985).

[10] B. Andersson, G. Gustafson, G. Ingelman, and T. Sjöstrand, Phys. Rep. 97, 31 (1983).

[11] G. Ingelman, A. Edin, and J. Rathsman, Comput. Phys. Commun. 101, 108 (1997).

[12] T. Sjöstrand, PYTHIA 5.7 and JETSET 7.4, Report No. CERNTH.7112/93 (unpublished); Comput. Phys. Commun. 82, 74 (1994).

[13] W. Buchmüller and S.H.H. Tye, Phys. Rev. D 24, 132 (1981).

[14] E. Braaten and T.C. Yuan, Phys. Rev. Lett. 71, 1673 (1993); Phys. Rev. D 52, 6627 (1995).

[15] E. Braaten and T.C. Yuan, Phys. Rev. D 50, 3176 (1994).

[16] P. Ernström and L. Lönnblad, Z. Phys. C 75, 51 (1997). 